\title{
Film Labels: A New Look
}

\author{
Tim B. Hunter
}

Every diagnostic image should be properly labeled. To improve the labeling of radiographs in the Department of Radiology at the University Medical Center, Tucson, Arizona, a special computer program was written to control the printing of the department's film flashcards. This program captures patient data from the hospital's radiology information system and uses it to create a film flashcard that contains the patient's name, hospital number, date of birth, age, the time the patient checked into the radiology department, and the date of the examination. The resulting film labels are legible and aesthetically pleasing. Having the patient's age and date of birth on the labels is a useful quality assurance measure to make certain the proper study has been performed on the correct patient. All diagnostic imaging departments should institute measures to assure their film labeling is as legible and informative as possible.

Copyright $\odot 1994$ by W.B. Saunders Company

$\mathbf{E}$ VERY DIAGNOSTIC image should be properly labeled. This is fundamental to good radiology and good patient care. There are several ways to label a radiograph. Holders containing small metallic numbers and letters can be placed on the corner of the film cassette. If properly exposed, they produce very legible labels. However, the information is often minimal because of the effort it takes to compose the label.

Large, separate individual letters and markers are very convenient for labeling such examinations as extremities and mammograms. Unfortunately, these may contain even less information than the holders and are often haphazardly spread across the radiographic cassette and the resulting radiographic image.

Gummed labels directly applied onto a radiograph work well and are useful for correcting errors made with film flashcard or metal labeling systems. Gummed labels with large, easily readable dates help to solve the problem of illegible dates. However, stick-on labels are not transparent (someone ought to look into this) and difficult to read if put on the nonviewing side of the radiograph.

Presently, many departments use some type of flashcard system. Patient information is typed or printed on a small card that is placed in a battery operated, hand held device which is then inserted into a corner of the film cassette.
The device flashes a pulse of light through the label, photographing the flashcard image onto the radiograph.

No matter the method used for radiograph labeling, the majority of the labels always seem to be upside down or backwards when the film is submitted for interpretation. Thus, out of the eight ways to put up a radiograph (correct, mirror image of correct placement, upside down, mirror image upside down, $90^{\circ}$ tilt to the right, $90^{\circ}$ tilt to the left, and mirror image of right and left tilt) only one or two at the most will be acceptable, and these do not always produce positioning of the label so that it will be easily visible. To solve this problem in our department, a special computer program, LABEL, was written to control the printing of our film flashcards.

\section{MATERIALS AND METHODS}

LABEL was written by Robert Morrison (PO Box 42372, Tucson, AZ 85734) in compiled BASIC and MUMPS to create a new font set containing routine, upside down, tilted, and mirror image characters. This program can either be used to capture data from one's radiology information system (RIS) or to control a small microcomputer system devoted to printing film flashcards and stick-on labels.

The program as written in MUMPS has been directly installed in our DECrad (IDX Corporation, South Burlington, VT) RIS. For this application, LABEL creates a special font set on a DEC LA 75 printer (Digital Equipment Corporation, Maynard, MA). In the future the program may be further adapted to control a laser printer.

\section{RESULTS AND DISCUSSION}

Whenever a patient checks into the radiology department for a study, the receptionist enters

From the Department of Radiology, Arizona Health Sciences Center, Tucson, $A Z$.

Address reprint requests to Tim B. Hunter, MD, Arizona Health Sciences Center, Department of Radiology, Tucson, $A Z$ 85724.

Copyright $\odot 1994$ by W.B. Saunders Company 0897-1889/94/0701-0003\$03.00/0 

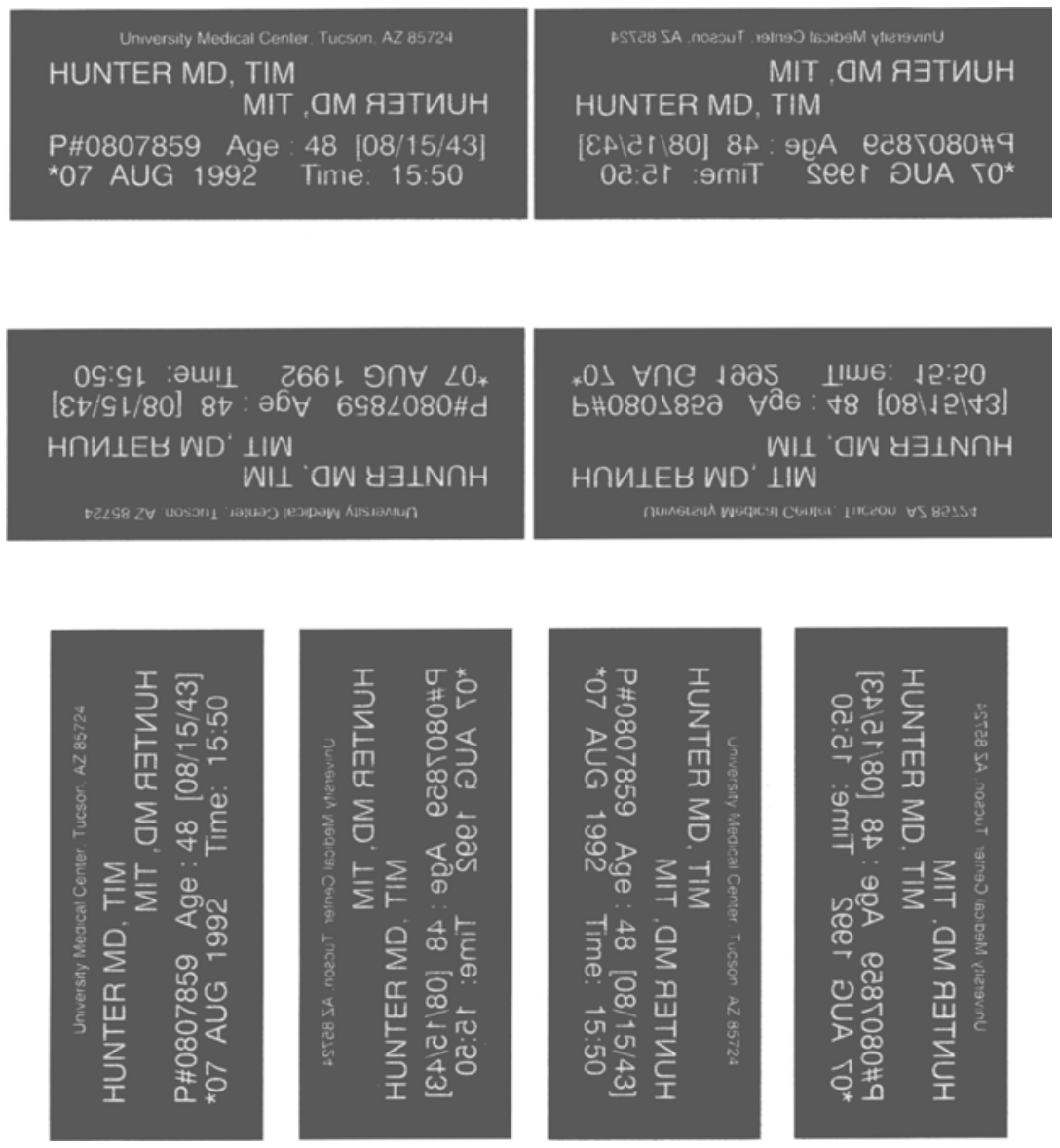

Fig 1. Film flashcard designed for legibility and quality control. From left to right, top to bottom, respectively, the figure shows the card placed in the following positions: correct, mirror image, upside down, mirror image upside down, $90^{\circ}$ left tilt, mirror image $90^{\circ}$ left tilt, $90^{\circ}$ right tilt, and mirror image $90^{\circ}$ right tilt. The first line lists the name and location of the institution. Lines 2 and 3 list the patient's name. Line 3 is a mirror image of line 2. Line 4 lists the patient's hospital number, age, and date of birth which is shown in parentheses. The patient's age is calculated from the date of birth. Line 5 shows the examination date and the time at which the flashcard was printed, rendered in military mode.

the patient's demographic information into our RIS system, which then instructs the DEC LA 75 printer to print a flashcard for the patient's radiographs. Because the flashcard has mirror image characters (Figs 1,2), the resulting label is readily legible no matter how a radiograph is placed on the viewbox.

The flashcard also has a built-in potential quality control/quality assurance mechanism to monitor the length of time the patient spends in the department. The time at which the flashcard was created is printed on the bottom line of the card next to the examination date. This usually represents the time at which the patient checked into the department. Okamoto Daylight ID Printers type Al (Okamoto Manufacturing Co,
Fig 2. Actual radiographic image of the film flashcard shown in Fig 1 . In the upper left hand corner, note the date and time the film was "flashed" before being placed in the film processor. In the example shown, the patient, a 48-year-old male, checked into the department on 7 August 1992 at 15:50 hours, and his radiograph was processed 2 minutes later at 15:52 hours.

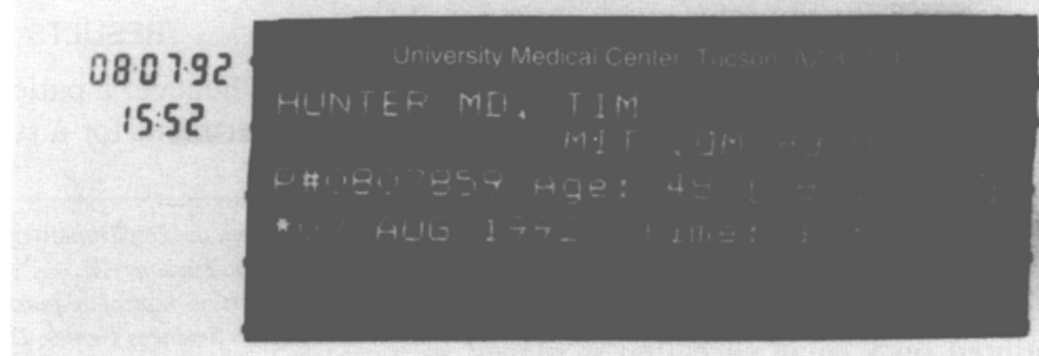


Ltd, Japan) attached to our Fuji FPM 9000 film processors (Fuji Medical Systems USA, Inc, Stamford, CT) expose the flashcard information onto each film before its development. The Okamoto printers also print in the upper left hand corner of the flashcard space on each film the date and time at which the film was "flashed" before being inserted into the film processor (Fig 2).

The difference between these two times ideally represents the time the patient spent in the department. In this regard, one has to be careful that the patient's flashcard, eg, was not printed the night before an early morning scheduled portable examination, or the patient's films were not saved for later batch processing after a morning's run of portable studies.

A very convenient feature of our flashcard system is the placement of the patient's hospital number, birthdate, and age on every label. The patient's age is calculated from his or her birthdate. Having the patient's exact age, birthdate, and hospital number on each radiograph also serves as a quality assurance measure to make certain the images presented for interpretation actually correspond to the patient and radiologic study requested by the patient's referring physician.

Film flashcards are relatively small (about $2.5 \times 6.0 \mathrm{~cm}$ in this case) and only so much information can be crowded onto them. A multitude of upside down and mirror image characters could be more confusing than help- ful. There has to be a reasonable balance between making the label legible in multiple positions versus jamming it with too many characters or extraneous patient information. This is a matter of judgement that will vary from medical center to medical center.

Digital studies, computed tomography (CT), magnetic resonance imaging (MRI), ultrasound, and nuclear medicine examinations are generally well labeled, if not overly so. The computer controlled equipment correctly orients the relevant patient information on each image. This does not mean that there is not room for considerable improvement. Typically, the images on CT, MRI, and ultrasound machines are crowded with much irrelevant, distracting information that could easily be saved for the header. Often, the actual image occupies $50 \%$ or less of the space reserved for each slice.

Manufacturers should create programs similar to LABEL. The use of more sophisticated software coupled with lasers printers offers the possibility of extremely clear, legible film labeling with near $100 \%$ consistency. In addition, manufacturers ought to greatly improve the poor labeling found on most CT, ultrasound, and MRI examinations. It is difficult to comprehend that a computer controlled device costing hundreds of thousands of dollars does not have a simple software package allowing the technologist or physician user to customize the image labeling at will. 